\title{
Infrared flux excesses from hot subdwarfs ${ }^{\star}$
}

\section{72 more objects s $^{\star \star}$}

\author{
A. Ulla ${ }^{1,2}$ and P. Thejll ${ }^{3}$ \\ 1 Universidade de Vigo, Departamento de Física Aplicada, Area de Física da Terra, Astronomía e Astrofísica, Facultade de \\ Ciencias, Campus Marcosende-Lagoas, Apartado Postal 874, E-36200 Vigo, Spain \\ 2 Instituto de Astrofísica de Canarias, E-38200 La Laguna, Spain \\ 3 Danish Meteorological Institute, Lyngbyvej 100, DK-2100 Copenhagen, Denmark
}

Received December 19, 1996; accepted March 20, 1998

\begin{abstract}
In our search, started in February, 1994, for $J H K$ excess fluxes among the hot subdwarf population as an indicator for the presence of binary companions, results for 72 more hot objects (=63 hot subdwarfs + 1 Horizontal Branch B star +7 white dwarfs +1 nonsubdwarf object) observed with the Carlos Sánchez CVF IR photometer (in June and October, 1994), are presented. The exact number of binary hot subdwarfs has gained renewed importance after the recent discovery of pulsators with G-F companions. The total number of candidates we propose may help to set some constraints; for example, out of 41 objects with excesses, 13 may have G-type binary companions. From our new sample, 14 discoveries of binary candidates have been found: BD+25 4655, Feige 108, HD 4539, HD 149382, HD 216135, KPD 2109+440, LSI+63 198, LSIV+10 9, LSV+22 38, PG 0011+221, PG 0116+242, PG 0314+103, PG 2151+100 and TON 139. Besides, 2 more from reanalysis of February, 1994, data $\mathrm{BD}+371977$ and BD+48 1777, may now be found to be IR excess candidates. Two suspected binaries, PB 8555 and SB 7, are also confirmed. By fitting Kurucz (1993) model spectra and assuming zero-age main sequence companions, we find upper limits on the subdwarf gravities. The distributions of upper limits on $\log (g)$, mostly between about 5.25 and 6.5, are nearly identical for both sdBs and sdOs.
\end{abstract}

Key words: surveys — stars: subdwarfs — binaries: spectroscopic — white dwarfs - infrared: stars

\footnotetext{
Send offprint requests to: A. Ulla, email address: ulla@uvigo.es * Based on observations made with the Carlos Sánchez Telescope operated on the island of Tenerife by the Instituto de Astrofísica de Canarias in the Spanish Observatorio del Teide.

${ }^{\star \star}$ Figure 1 is only available in the electronic version of the paper (http://www.edpsciences.com)
}

\section{Introduction}

Thejll et al. (1995; hereafter Paper I) discussed the possible origin for excess infrared fluxes detected from the direction of a hot subdwarf star (sd). They presented a set of $J H K$ measurements for 27 hot objects: 23 hot sds, GD274 -which the authors reclassify as sd+K3-K8, and 3 white dwarfs (WDs). An analysis was also presented for the, then 24, hot subdwarfs. After considering a wind expelled from the hot atmosphere as the source of the excess IR radiation, the authors concluded that the most likely explanation is the presence of a cool stellar companion giving rise to the observed excess. With the aid of comprehensive literature and database searches, several well known cases were corroborated, suspected cases confirmed and new discoveries uncovered.

The point in studying companions to hot subdwarfs is that we can learn things about the hot subdwarfs that are not easy to observe directly due to the problems of modeling hot atmospheres and the relatively large distances to these stars that preclude some astrometric analysis for the majority of these stars. Parallaxes are hard to measure but some are expected from the HIPPARCOS data for a small number of close stars. Proper motion analysis has been performed by Thejll et al. (1997) Spectral analyses of hot subdwarf B stars (e.g. Saffer et al. 1994) and sdO stars (Thejll et al. 1994a) exist. Our work presents an investigation on the properties of the companion stars, and the hot subdwarf properties that can be inferred from these.

Following a similar approach to that in Paper I, the results presented here relate to 72 more stars (63 hot sds; 1 Horizontal Branch B (HBB) star - PG 0342+026; 7 WDs; and the non-sd object PHL 382 - Kilkenny, private communication) observed with the same telescope and instrumentation. In the present paper, among other means of analysis, they are analysed following the methods described in Paper I but the UV, optical and IR fluxes are 
Table 1. List of observed objects. Most common names, spectral type, coordinates (Epoch 1950.0 or stated otherwise), temperatures and gravities available in the literature, or estimated in this paper from published photometry and UV fluxes, together with source references for program stars, are given. Notes: $\star=2000.0$ coordinates; $1=$ This work; pI=Thejll et al. (1995); A94=Allard et al. (1994); BH95=Bauer \& Husfeld (1995); Bo94=Bowyer et al. (1994); D90=Dreizler et al. (1990); D93=Dreizler (1993); G80=Giddings (1980); GS74=Greenstein \& Sargent (1974); H86=Heber (1986); HB95=Hurwitz \& Bowyer (1995); HL86=Heber \& Langhans (1986); Je92=Jeffery et al. (1992); Ji94=Jiménez et al. (1994); K=Kilkenny, private communication; Ma95=Marilli et al. (1995); Mo90=Moehler et al. (1990); N93a=Napiwotzki (1993a); N93b=Napiwotzki (1993b); P70=Peterson (1970); S94=Saffer et al. (1994); T93=Theissen et al. (1993); T95=Theissen et al. (1995); Th96=Thejll, Husfeld \& Saffer (unpublished work); To70=Tomley (1970); V91=Viton et al. (1991); Ve93=Vennes et al. (1993); Ve94=Vennes et al. (1994); Wo96=Wolff et al. (1996). Temperatures in parenthesis indicate that there is not enough data to asses the temperature but that an estimate has been made on the basis of literature spectral classes - thus any un-analyzable sdO is set to $40000 \mathrm{~K}$ while all un-analyzable sdB or sd are set to 20000 K. HD 113001 and GD 274 were observed in February (1994)

\begin{tabular}{|c|c|c|c|c|c|c|c|c|c|c|}
\hline Object & Sp. type & $\mathrm{hh}$ & $\mathrm{mm}$ & SS & $\overline{0}$ & & & $\overline{T_{\text {eff }}}$ & $\log (g)$ & ref. \\
\hline \multicolumn{11}{|c|}{ hot sds/HBB previously unknown as binaries } \\
\hline $\mathrm{BD}+254655$ & $\mathrm{sdO}$ & 21 & 57 & 25.05 & +26 & 11 & 34.8 & 42 & 6.7 & $\mathrm{P} 70$ \\
\hline $\mathrm{BD}+393226$ & sdOp & 17 & 44 & 52.66 & +39 & 20 & 11.8 & 45 & 5.5 & G80 \\
\hline Feige 11 & $\mathrm{sdB}$ & 01 & 01 & 46 & +03 & 57 & 35 & 28.4 & 5.63 & S94 \\
\hline Feige 65 & $\operatorname{sdB}$ & 12 & 33 & 27 & +42 & 39 & 13 & 26.5 & 5.6 & S94 \\
\hline Feige 108 & sdB & 23 & 16 & 12 & -01 & 50 & 35 & 34.5 & 6.01 & $\mathrm{~S} 94, \star$ \\
\hline Feige 110 & $\mathrm{sdO}$ & 23 & 17 & 23.5 & -05 & 26 & 22 & 42 & 5.9 & Th96 \\
\hline HD 4539 & $\operatorname{sdB}$ & 00 & 47 & 29.2 & +09 & 58 & 56.4 & $25-30,27$ & $5,5.46$ & $\mathrm{HL}, \mathrm{S} 94, \star$ \\
\hline HD 149382 & sdB & 16 & 31 & 45.25 & -03 & 54 & 40.9 & 34.2 & 5.89 & $\mathrm{~S} 94$ \\
\hline HD 216135 & sdB/sdBk/sdB5 & 22 & 47 & 50.0 & -13 & 34 & 20 & 15 & - & 1 \\
\hline HZ 3 & sdO & 03 & 53 & 32 & +10 & 46 & 02 & 46 & 7: & 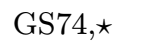 \\
\hline $\mathrm{HZ} 44$ & $\mathrm{sdO} / \mathrm{sdO} 8 \mathrm{p}$ & 13 & 21 & 18.8 & +36 & 23 & 37 & 40 & $5.3-5.7$ & GS74, P70 \\
\hline KPD2109+440 & $\mathrm{sdB}$ & 21 & 09 & 56 & +44 & 01 & 36 & 25 & - & 1 \\
\hline LSI+63 198 & sdO & 03 & 24 & 42 & +63 & 54 & 20.1 & 34 & 5.4 & GS74 \\
\hline LSIV-14 116 & $\mathrm{sdOB} / \mathrm{sdO} \mathrm{He}$ & 20 & 54 & 53 & -14 & 37 & 26 & 35 & - & 1 \\
\hline LSIV +00 21 & $\mathrm{sdB}$ & 20 & 28 & 45.3 & +00 & 55 & 12 & 34 & - & 1 \\
\hline LSIV+109 & $\mathrm{sdO}$ & 20 & 40 & 36 & +10 & 23 & 19.8 & 45 & 5.6 & D93 \\
\hline $\mathrm{LSV}+2238$ & sdO & 06 & 13 & 12 & +22 & 46 & 54 & $(40)$ & - & 1 \\
\hline PG $0011+221$ & $\mathrm{sd}$ & 00 & 11 & 43.7 & +22 & 07 & 41 & 30 & - & 1 \\
\hline PG $0116+242$ & sd & 01 & 16 & 46.6 & +24 & 09 & 48 & 14 & - & 1 \\
\hline PG $0122+214$ & sd & 01 & 22 & 44.4 & +21 & 21 & 13 & 19 & - & 1 \\
\hline PG $0133+114$ & $\mathrm{sdB} / \mathrm{sd}$ & 01 & 33 & 47 & +11 & 24 & 14 & 26 & 4.9 & Mo90 \\
\hline PG $0215+183$ & $\mathrm{sdB}$ & 02 & 15 & 28.6 & +18 & 18 & 04 & 22 & - & 1 \\
\hline PG $0242+132$ & $\mathrm{sdB}$ & 02 & 42 & 54.7 & +13 & 13 & 27 & 31.2 & 5.65 & S94 \\
\hline PG $0314+103$ & $\mathrm{sdB}$ & 03 & 14 & 59.2 & +10 & 19 & 57 & 18 & - & 1 \\
\hline PG $0342+026$ & $\mathrm{HBB}$ & 03 & 42 & 58 & +02 & 38 & 36 & 26.2 & 5.67 & S94 \\
\hline PG $0856+121$ & sdB & 08 & 56 & 18.8 & +12 & 08 & 06 & 26.4 & 5.73 & S94 \\
\hline PG $1338+481$ & $\mathrm{sdB}$ & 13 & 38 & 05.7 & +48 & 07 & 41 & 25 & - & 1 \\
\hline PG $1409+605$ & sdB-O & 14 & 09 & 31.5 & +60 & 28 & 29 & 22 & - & 1 \\
\hline PG $2111+023$ & $\mathrm{HBB} / \mathrm{sdB}$ & 21 & 11 & 10.5 & +02 & 20 & 43 & 15 & - & 1 \\
\hline PG $2128+146$ & sd & 21 & 28 & 55.7 & +14 & 36 & 11 & 14 & - & 1 \\
\hline PG $2151+100$ & $\mathrm{sd}$ & 21 & 51 & 21.8 & +10 & 01 & 14 & 27 & - & 1 \\
\hline PG $2158+082$ & $\mathrm{sdO} \mathrm{He} / \mathrm{sdO}$ & 21 & 58 & 25.8 & +08 & 14 & 04 & 67 & 5.5 & D90 \\
\hline PG $2159+051$ & sd & 21 & 59 & 20.3 & +05 & 07 & 47 & 15 & - & 1 \\
\hline PG $2218+020$ & sdB-O & 22 & 18 & 52 & +02 & 01 & 07 & 20 & - & 1 \\
\hline PG $2318+239$ & sd & 23 & 18 & 36.5 & +23 & 54 & 08 & 16 & - & 1 \\
\hline PG $2321+214$ & $\operatorname{sdO}(\mathrm{D}) / ?$ & 23 & 21 & 57.6 & +21 & 22 & 23 & 43 & - & Th96 \\
\hline PG $2345+241$ & sdB-O & 23 & 45 & 50.3 & +24 & 06 & 25 & 20 & - & 1 \\
\hline PG $2352+181$ & $\mathrm{sdO} \mathrm{He} / \mathrm{sdO}(\mathrm{B})$ & 23 & 52 & 44.2 & +18 & 03 & 36 & 55 & 6.4 & Th96 \\
\hline PHL 25 & $\mathrm{sdB}$ & 21 & 31 & 58 & -17 & 18 & 43 & 19 & - & $1, \star$ \\
\hline PHL 678 & $\mathrm{sdB} / \mathrm{sdB}-\mathrm{O}$ & 00 & 04 & 59 & +13 & 19 & 10 & 25 & 4.5 & Mo90 \\
\hline PHL 2726 & $\mathrm{~B} / \mathrm{sd}$ & 00 & 09 & 54 & +03 & 37 & 46 & 15 & - & 1 \\
\hline SB 395 & $\mathrm{sdB}$ & 00 & 56 & 48 & -18 & 33 & 00.4 & 15 & - & 1 \\
\hline TON 139 & $\mathrm{sdB}$ & 12 & 53 & 39.6 & +28 & 23 & 31 & 18 & - & 1 \\
\hline TON 209 & $\mathrm{sdB} / \mathrm{sdOp}$ & 14 & 33 & 05.0 & +23 & 58 & 31 & 29.6 & 5.57 & S94 \\
\hline TON 245 & $\mathrm{sdB}$ & 15 & 38 & 24 & +26 & 57 & 00.0 & 25.2 & 5.30 & S94 \\
\hline TON 788 & $\mathrm{sdB}$ & 15 & 12 & 21 & +24 & 21 & 43 & 25 & - & 1 \\
\hline UVO0653-23 & sdB & 06 & 53 & 12.7 & -23 & 28 & 21 & 15 & - & 1 \\
\hline
\end{tabular}


Table 2. List of observed objects (continuation). $\dagger=\mathrm{S} 94$ reports $29700 \mathrm{~K}$ and $\log (g)=4.6$ but that would make the contribution from the companion star so dominant in the optical range that the spectral analysis by S94 is meaningless

\begin{tabular}{|c|c|c|c|c|c|c|c|c|c|c|}
\hline Object & Sp. type & hh & $\mathrm{mm}$ & ss & & & & $T_{\text {eff }}$ & $\log (g)$ & ref. \\
\hline \multicolumn{11}{|c|}{ known or suspected hot sd binaries } \\
\hline $\mathrm{BD}-35357$ & sdOB+G8III/e.bin. & 21 & 58 & 00.87 & -02 & 58 & 52.7 & $35-40$ & - & Ma95 \\
\hline $\mathrm{BD}-75977$ & $\mathrm{sdOB}+\mathrm{F} 8-\mathrm{G} 0 \mathrm{~V}$ & 23 & 15 & 11.6 & -06 & 44 & 56 & 31 & - & V91 \\
\hline $\mathrm{BD}-11162$ & $\mathrm{sdO}+\mathrm{G}$ & 00 & 49 & 43.8 & -10 & 56 & 14 & 35 & 5.9 & 1 \\
\hline $\mathrm{BD}+284211$ & $\mathrm{sdO}+\mathrm{G}$ & 21 & 48 & 57.5 & +28 & 37 & 44 & 82 & 6.2 & N93a \\
\hline $\mathrm{BD}+293070$ & $\mathrm{sdOB}+\mathrm{F}$ & 17 & 36 & 22.3 & +29 & 10 & 29 & 18 & - & 1 \\
\hline GD 274 & $\mathrm{sd}+\mathrm{K} 3-\mathrm{K} 8$ & 01 & 04 & 14 & +50 & 54 & 25 & $18.5-25$ & $4.5-7$ & $\mathrm{pI}$ \\
\hline HD 45166 & $\mathrm{~B} 8 \mathrm{~V}+(\mathrm{sdO} / \mathrm{qWR})$ & 06 & 23 & 36.0 & +08 & 00 & 16 & 40 & - & 1 \\
\hline HD 113001 & $\mathrm{sdO}+\mathrm{F} / \mathrm{v} \cdot \mathrm{bin}$. & 12 & 58 & 05 & +36 & 01 & 32 & $41,41-50$ & $5.7,6.0$ & To70,pI \\
\hline HD 185510 & sdB+K0III-IV & 19 & 36 & 58.42 & -06 & 10 & 44.8 & 25 & $\sim 6$ & Je92 \\
\hline MRK509C & $\mathrm{sdO}+\mathrm{K} 9-\mathrm{M} 1$ & 20 & 43 & 59.0 & -10 & 47 & 42 & 42.5 & - & Ji94 \\
\hline PB 8555 & $\mathrm{sd}+\mathrm{F} ?$ & 01 & 07 & 44 & -14 & 24 & 00.0 & $(20)$ & - & 1 \\
\hline PG $2102+037$ & sdO/comp. & 21 & 02 & 36.3 & +03 & 42 & 43 & $\geq 80$ & - & Th96 \\
\hline PG $2110+127$ & $\mathrm{sdB}+\mathrm{G} 6 \mathrm{IV}$ & 21 & 10 & 56.4 & +12 & 44 & 44 & $33.7,30$ & $5.33,5.0$ & S94,T93 \\
\hline PG $2118+126$ & $\mathrm{sdB}+\mathrm{K} 2$ & 21 & 18 & 37.9 & +12 & 38 & 03 & 26.5 & - & A94 \\
\hline PG 2148+095 & $\mathrm{sdB}+\mathrm{K} 3$ & 21 & 48 & 41.2 & +09 & 30 & 39 & 25 & - & 1 \\
\hline PG 2219+094 & $\mathrm{sdB}+(\mathrm{Ca} \mathrm{K})$ & 22 & 19 & 30.0 & +09 & 22 & 21 & 21 & - & $1, \dagger$ \\
\hline PG 2229+099 & $\mathrm{HBB} / \mathrm{sdB}+(\mathrm{Ca} \mathrm{K})$ & 22 & 29 & 39.2 & +09 & 58 & 58 & 18.1 & 3.9 & S94 \\
\hline PHL 1079 & $\mathrm{sdB}+\mathrm{G} 8 \mathrm{IV}$ & 01 & 35 & 48 & +03 & 23 & 00.0 & 30 & 5.25 & T95 \\
\hline SB 7 & $\mathrm{sdB} / \mathrm{bin} ?$ & 00 & 00 & 48 & -16 & 37 & 00.0 & 55 & - & $\mathrm{H} 86$ \\
\hline \multicolumn{11}{|l|}{ CSPN } \\
\hline BD-13 842 & CSPN & 04 & 11 & 57.0 & -12 & 51 & 42 & 70 & 4.6 & BH95 \\
\hline $\mathrm{BD}+332642$ & CSPN & 15 & 50 & 02.0 & +33 & 05 & 56 & & & N93b \\
\hline \multicolumn{11}{|c|}{ WDs and others } \\
\hline Feige 24 & $\overline{\mathrm{DA}+\mathrm{dM}}$ & 02 & 32 & 31 & +03 & 30 & 06 & & & Ve94 \\
\hline G191-B2B & DAw+sdK4pec & 05 & 01 & 31 & +52 & 44 & 48 & & & HB95 \\
\hline GD 246 & DAw & 23 & 12 & 23 & +10 & 46 & 07 & & & $\star, \mathrm{Ve} 93$ \\
\hline J0633+104 & DA & 06 & 33 & 53 & +10 & 42 & 06 & & & $\star$, Bo94 \\
\hline $\mathrm{J} 2013+400$ & $\mathrm{DA}+\mathrm{dM} 4$ & 20 & 13 & 08 & +40 & 02 & 36 & & & $\star, B o 94$ \\
\hline $\mathrm{J} 2210-300$ & WD/vis. bin & 22 & 10 & 29 & -30 & 05 & 48 & & & $\star$, Bo94 \\
\hline PG $1234+482$ & DA & 12 & 34 & 23 & +48 & 11 & 54 & & & Wo96 \\
\hline PHL 382 & non-sd & 22 & 40 & 30 & -15 & 06 & 46 & 19 & - & $\mathrm{K}$ \\
\hline
\end{tabular}

interpreted with the aid of Kurucz spectral models - in Paper I black body (BB) functions were used. Objects in Paper I are therefore reanalyzed and the corresponding results included here. Out of the $99(=27+72)$ stars observed with the CST in 1994, the total number of hot subdwarf/HBB stars studied, i.e., $88(=23+$ GD 274 , from February, + 63 + PG 0342+026, from June and October), basically corresponds to the total possible number of this kind of objects observable, as extracted from Kilkenny et al. (1988), given the limiting magnitude of the instrument employed $(\sim 13.5 \mathrm{mag}$ in $K)$. This total sample is, to our knowledge, the most complete $J H K$ hot subdwarf catalogue available to date. Most of these targets are observed at IR wavelengths for the first time. Tables 1 and 2 present the 72 objects added in the present work.

Our paper consists of a part presenting the $J H K$ observations and two analysis parts. The reader is referred to Paper I for details of these methods. The JHK observations and the reduction procedure are discussed in Sect. 2. Compilation of optical and UV data, and treatment of the latter, is described in Sect. 3. The detection and ex- traction of excess fluxes using estimates of the $T_{\text {eff }}$ of the hot subdwarf is discussed in Sect. 4. We then proceed in Sect. 5 to analyze the excesses and calculate the gravities of the hot subdwarfs assuming that stellar companions are zero-age main sequence (ZAMS) stars. Section 6 presents a summary and discussions.

\section{IR observations and data calibration}

The JHK photometry was obtained for the objects in Tables 1 and 2 with the $1.5 \mathrm{~m}$ Carlos Sánchez Telescope (CST) (Arribas \& Martínez-Roger 1987) at the Observatorio del Teide (Tenerife), in June and October, 1994. Targets were chosen as in Paper I, with less emphasis on reproducing measurements in Probst (1983). A few WD targets were included. Their analysis will be presented elsewhere (Vennes et al. 1998). GD 274 was observed nightly, in October 1994, to check for reproducibility of results, and the distribution of the measurements are fully consistent with the photometric errors of each measurement. 
Table 3. Results from the June 1994 run at the CST. Each entry is the result of averaging of 3 or more integrations. When several entries are given, separate integration-series were performed. ${ }^{a}$ after an object's name means weighted average of all its $J H K$ measurements as given in Tables 3, 4 and 5

\begin{tabular}{|c|c|c|c|c|c|c|}
\hline Object & $J$ & $\sigma_{J}$ & $H$ & $\overline{\sigma_{H}}$ & $K$ & $\overline{\sigma_{K}}$ \\
\hline \multicolumn{7}{|c|}{ hot sds previously unknown as binaries } \\
\hline $\mathrm{BD}+393226$ & 10.80 & 0.05 & 10.99 & 0.04 & 11.11 & 0.03 \\
\hline Feige 65 & 12.48 & 0.23 & 12.65 & 0.17 & 12.69 & 0.26 \\
\hline HD 149382 & 9.39 & 0.03 & 9.35 & 0.02 & 9.36 & 0.03 \\
\hline HZ 44 & 12.20 & 0.02 & 12.85 & 0.02 & 12.83 & 0.02 \\
\hline HZ 44 & 12.20 & 0.16 & 12.60 & 0.12 & 12.28 & 0.17 \\
\hline $\mathrm{HZ} 44^{a}$ & 12.20 & 0.02 & 12.84 & 0.02 & 12.82 & 0.02 \\
\hline LSIV+00 21 & 12.74 & 0.34 & 12.72 & 0.18 & 12.86 & 0.27 \\
\hline $\mathrm{LSIV}+0021$ & 13.88 & 0.73 & 12.79 & 0.18 & 12.59 & 0.27 \\
\hline $\mathrm{LSIV}+0021^{a}$ & 12.94 & 0.31 & 12.76 & 0.13 & 12.72 & 0.19 \\
\hline LSIV+10 9 & 11.15 & 0.03 & 10.98 & 0.05 & 10.90 & 0.05 \\
\hline PG $1338+481$ & 14.85 & 1.67 & 14.05 & 0.68 & 14.68 & 1.57 \\
\hline PG $1409+605$ & 13.80 & 0.50 & 13.29 & 0.19 & 13.90 & 0.65 \\
\hline PG 2151+100 & 12.39 & 0.57 & 11.49 & 0.06 & 11.23 & 0.06 \\
\hline PG $2151+100$ & 11.86 & 0.08 & 11.48 & 0.04 & 11.40 & 0.11 \\
\hline PG 2218+020 & 14.35 & 0.38 & 14.63 & 0.76 & 15.05 & 1.63 \\
\hline TON 139 & 12.10 & 0.15 & 11.92 & 0.08 & 11.93 & 0.16 \\
\hline TON 209 & 13.46 & 0.53 & 13.34 & 0.28 & 13.68 & 0.82 \\
\hline TON 245 & 13.75 & 0.35 & 14.32 & 0.42 & 14.85 & 0.59 \\
\hline TON 788 & 13.81 & 0.30 & 13.78 & 0.24 & 14.72 & 0.96 \\
\hline \multicolumn{7}{|c|}{ known or suspected hot sd binaries } \\
\hline $\mathrm{BD}-35357$ & 7.28 & 0.01 & 6.74 & 0.01 & 6.59 & 0.01 \\
\hline $\mathrm{BD}+284211$ & 11.26 & 0.15 & 11.17 & 0.09 & 11.28 & 0.08 \\
\hline $\mathrm{BD}+284211$ & 11.23 & 0.11 & 11.36 & 0.14 & 11.75 & 0.36 \\
\hline $\mathrm{BD}+284211^{a}$ & 11.24 & 0.09 & 11.23 & 0.08 & 11.30 & 0.08 \\
\hline $\mathrm{BD}+293070$ & 9.74 & 0.01 & 9.55 & 0.01 & 9.54 & 0.03 \\
\hline HD 113001 & 9.02 & 0.02 & 8.83 & 0.01 & 8.80 & 0.01 \\
\hline HD 113001 & 9.07 & 0.01 & 8.87 & 0.01 & 8.84 & 0.02 \\
\hline HD $113001^{a}$ & 9.06 & 0.01 & 8.85 & 0.01 & 8.81 & 0.01 \\
\hline HD 185510 & 6.09 & 0.02 & 5.52 & 0.01 & 5.38 & 0.01 \\
\hline HD 185510 & 6.10 & 0.02 & 5.53 & 0.01 & 5.37 & 0.01 \\
\hline HD 185510 & 6.23 & 0.01 & 5.69 & 0.03 & 5.43 & 0.01 \\
\hline $\mathrm{HD} 185510^{a}$ & 6.18 & 0.01 & 5.53 & 0.01 & 5.39 & 0.01 \\
\hline MRK509C & 12.67 & 0.45 & 12.20 & 0.22 & 12.00 & 0.20 \\
\hline MRK509C & 12.90 & 0.34 & 13.48 & 0.32 & 14.03 & 1.26 \\
\hline MRK509C & 13.95 & 0.74 & 13.62 & 0.29 & 13.22 & 0.49 \\
\hline MRK509C $^{a}$ & 12.95 & 0.26 & 12.83 & 0.15 & 12.25 & 0.20 \\
\hline PG 2102+037 & 14.92 & 2.79 & 13.10 & 0.24 & 13.70 & 0.97 \\
\hline PG $2110+127$ & 12.42 & 0.29 & 11.99 & 0.12 & 12.23 & 0.21 \\
\hline PG $2110+127$ & 12.17 & 0.12 & 12.11 & 0.12 & 12.11 & 0.12 \\
\hline \multicolumn{7}{|l|}{ CSPN } \\
\hline $\mathrm{BD}+332642$ & 11.09 & 0.05 & 11.08 & 0.02 & 11.11 & 0.07 \\
\hline \multicolumn{7}{|l|}{ DA WD } \\
\hline PG $1234+482$ & 14.24 & 0.68 & 14.83 & 1.19 & 15.80 & 3.97 \\
\hline
\end{tabular}

Table 4. Results from the October 1994 run at the CST. Notation is as in previous tables

\begin{tabular}{lrrrrrr}
\hline Object & $J$ & $\sigma_{J}$ & $H$ & $\sigma_{H}$ & $K$ & $\sigma_{K}$ \\
\hline \multicolumn{7}{l}{ hot sds previously unknown as binaries } \\
\hline BD +25 4655 & 10.39 & 0.02 & 10.52 & 0.01 & 10.58 & 0.03 \\
Feige 11 & 12.78 & 0.14 & 12.98 & 0.37 & 13.65 & 1.07 \\
Feige 108 & 13.63 & 0.15 & 13.01 & 0.25 & 12.88 & 0.32 \\
Feige 110 & 12.40 & 0.07 & 12.80 & 0.09 & 12.65 & 0.10 \\
HD 4539 & 10.82 & 0.04 & 10.93 & 0.03 & 10.96 & 0.03 \\
HD 216135 & 10.29 & 0.04 & 10.41 & 0.04 & 10.46 & 0.06 \\
HZ 3 & 13.21 & 0.44 & 13.59 & 0.34 & 13.55 & 0.48 \\
KPD2109+440 & 10.04 & 0.05 & 8.90 & 0.02 & 8.59 & 0.01 \\
LSI+63 198 & 7.30 & 0.02 & 6.64 & 0.01 & 6.50 & 0.02 \\
LSIV-14 116 & 12.74 & 0.27 & 14.20 & 0.69 & 14.36 & 4.12 \\
LSV+22 38 & 8.29 & 0.01 & 7.94 & 0.01 & 7.74 & 0.01 \\
LSV+22 38 & 8.45 & 0.04 & 8.10 & 0.04 & 7.93 & 0.04 \\
LSV+22 38 & 8.47 & 0.02 & 8.07 & 0.03 & 7.88 & 0.03 \\
LSV+22 38 & 8.33 & 0.01 & 7.96 & 0.01 & 7.76 & 0.01 \\
PG 0011+221 & 12.91 & 0.14 & 13.05 & 0.12 & 13.31 & 0.26 \\
PG 0116+242 & 10.88 & 0.02 & 10.57 & 0.02 & 10.46 & 0.05 \\
PG 0122+214 & 12.97 & 0.16 & 13.50 & 0.24 & 12.85 & 0.30 \\
\hline
\end{tabular}

known or suspected hot sd binaries

\begin{tabular}{lrrrrrr}
\hline BD-7 5977 & 8.97 & 0.02 & 8.48 & 0.01 & 8.38 & 0.01 \\
BD-11 162 & 10.81 & 0.03 & 10.64 & 0.03 & 10.61 & 0.03 \\
GD 274 & 11.33 & 0.07 & 10.93 & 0.03 & 10.88 & 0.04 \\
GD 274 & 11.26 & 0.07 & 10.96 & 0.03 & 10.79 & 0.07 \\
GD 274 & 11.27 & 0.09 & 10.94 & 0.04 & 10.84 & 0.06 \\
GD 274 & 11.30 & 0.02 & 10.94 & 0.02 & 10.97 & 0.04 \\
GD 274 & 11.30 & 0.10 & 10.96 & 0.04 & 10.85 & 0.04 \\
GD 274 & 11.35 & 0.05 & 11.02 & 0.02 & 10.88 & 0.04 \\
GD 274 & 11.37 & 0.12 & 10.89 & 0.05 & 11.19 & 0.23 \\
GD 274 & 11.41 & 0.09 & 10.92 & 0.05 & 10.74 & 0.07 \\
GD 274 & 11.31 & 0.02 & 10.96 & 0.01 & 10.88 & 0.02 \\
HD 45166 & 10.18 & 0.03 & 10.12 & 0.03 & 10.00 & 0.05 \\
PB 8555 & 11.30 & 0.04 & 11.07 & 0.03 & 10.94 & 0.04 \\
\hline CSPN & & & & & & \\
\hline BD-13 842 & 11.16 & 0.02 & 11.36 & 0.06 & 10.83 & 0.08 \\
\hline WDs and others & & & & & & \\
\hline Feige 24 & 11.17 & 0.07 & 10.68 & 0.03 & 10.45 & 0.09 \\
G191-B2B & 12.48 & 0.11 & 12.56 & 0.11 & 12.48 & 0.16 \\
GD 246 & 13.42 & 0.13 & 14.35 & 0.47 & 14.69 & 1.01 \\
J0633+104 & 14.03 & 0.86 & 15.93 & 1.36 & 16.22 & 5.95 \\
J2013+400 & 15.39 & 2.39 & 14.12 & 0.40 & 13.13 & 0.31 \\
J2210-300 & 12.55 & 0.14 & 12.43 & 0.15 & 12.16 & 0.27 \\
\hline & & & & & &
\end{tabular}


Table 5. Results from the October 1994 run at the CST (continuation)

\begin{tabular}{|c|c|c|c|c|c|c|}
\hline Un & $J$ & $\sigma_{J}$ & $H$ & $H$ & $K$ & $\sigma_{K}$ \\
\hline \multicolumn{7}{|c|}{ hot sds/HBB previously unknown as binaries } \\
\hline PG 0133+114 & 2.77 & 0.10 & 12.88 & 0.16 & 12.40 & 0.19 \\
\hline $33+$ & .78 & 0.06 & 4.68 & 0.13 & 12.72 & 0 \\
\hline PG 0133+114 & 13.08 & 0.41 & 13.15 & 0.47 & 12.37 & 0.64 \\
\hline $\mathrm{PG} 0133+114^{a}$ & 12.78 & 0.51 & 3 & 0.10 & 12 & 0.0 \\
\hline PG 0 & 13.50 & 0.25 & 13.97 & 0.26 & 14.12 & 0.54 \\
\hline $12+$ & 13.69 & 0.51 & 3.23 & 0.27 & 1280 & 1.30 \\
\hline PG $0242+132$ & 13.34 & 0.60 & 13.79 & 0.37 & 13.03 & 1.44 \\
\hline $\mathrm{PG} 0242+132^{a}$ & $1:$ & 0 & 13.42 & 0 & 13 & 0 \\
\hline $\mathrm{P}$ & 12.49 & 0.12 & 11.91 & 0.05 & 11.65 & 0.08 \\
\hline PG $0342+026$ & 11.67 & 8 & 11.89 & 0.13 & 11 & 0.20 \\
\hline $\mathrm{P}$ & 13.42 & 0.44 & 13.59 & 0.22 & 13.84 & 0.55 \\
\hline 10 & 13.26 & 0.42 & 13.17 & 0.19 & 14.35 & 3.60 \\
\hline $2128+146$ & 13.16 & 0.21 & 13.50 & 0.15 & 14.11 & 1.56 \\
\hline PG $2151+100$ & 12.60 & 0.08 & 12.50 & 0.08 & 13.67 & 0.75 \\
\hline $\mathrm{PG} 2151+100^{a}$ & 12.23 & 0.06 & 11.63 & 0.03 & 11.28 & 0.05 \\
\hline 10 & 12.89 & 2 & 13. & 0.35 & 6 & 1.35 \\
\hline $150+051$ & 13.14 & 0.17 & 13.07 & 0.15 & 13.68 & 0.56 \\
\hline & 4 & 7 & 8 & 0 & 0 & 54 \\
\hline & 14.17 & 0.48 & 14.26 & 0.31 & 14.04 & 0.31 \\
\hline 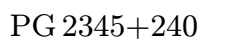 & 1 & 2 & 0 & 0.20 & & \\
\hline $252+181$ & 13.36 & 0.68 & 15.09 & 1.67 & 12.83 & 1.05 \\
\hline & 12.38 & 0.08 & 12 & 0.08 & 0 & 0.30 \\
\hline & 13.06 & 0.19 & 13.13 & 0.14 & 14.34 & 1.35 \\
\hline 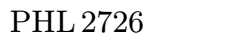 & 13.21 & 0.27 & 13.15 & 0.13 & 13.95 & 0.72 \\
\hline S & 12.89 & 0.14 & 13.03 & 0.11 & 13.23 & 0.23 \\
\hline 8000 & 3 & 0.03 & 07 & 0.04 & 10.08 & 0.05 \\
\hline \multicolumn{7}{|c|}{ known or suspected hot sd binaries } \\
\hline $0+127$ & 12.29 & 0.08 & 12.09 & 0.07 & 12 & 0.14 \\
\hline PG $2110+12$ & 1 & 0 & t & 0.05 & $1:$ & 0.08 \\
\hline & 12.71 & 0.11 & 12.69 & 0.10 & 12.66 & 0.18 \\
\hline $10 \mathrm{cor}$ & 12.18 & 0.11 & 12.34 & 0.17 & 12.06 & 0.4 \\
\hline PG 2219+094 & 12.21 & 0.09 & 12.24 & 0.06 & 12.26 & 0.14 \\
\hline & 13.39 & 0.16 & 13.61 & 0.17 & 13.34 & 0.43 \\
\hline & 12.55 & 0.17 & 12.23 & 0.06 & 12.04 & 0.08 \\
\hline SB 7 & 2.79 & 0.31 & 12.43 & 0.07 & 12.54 & 0.18 \\
\hline \multicolumn{7}{|l|}{ non-sd } \\
\hline PHL 3 & 1.53 & 0.05 & 11.62 & 0.05 & 11.64 & 0.08 \\
\hline
\end{tabular}

Table 6. Table of large-aperture $I U E$ spectra used in this work. "SWP", "LWP" and "LWR" respectively indicate spectra taken with the short and long wavelength primary, and redundant cameras. Results of reddening analysis of suitable $I U E$ spectra are also included. In spectra where reddening is not readily apparent an upper limit of $E(B-V) \leq 0.025$ is assigned. The uncertainties given are estimated visually and correspond roughly to 2 or 3 sigma intervals. For PHL 678 only an approximate value can be given for the large reddening for smaller values the " $2200 \AA$ bump" is still very clear and for larger values over-correction takes place, yet the correction at $E(B-V)=0.2$ is not perfect

\begin{tabular}{|c|c|c|c|}
\hline Object & $E(B-V)$ & SWP & LWP/LWR \\
\hline \multicolumn{4}{|c|}{ hot sds/HBB previously unknown as binaries } \\
\hline \multicolumn{2}{|c|}{$\mathrm{BD}+254655 \leq 0.025$} & 05640,01651 & 03387,04890 \\
\hline \multicolumn{2}{|c|}{$\mathrm{BD}+393226 \approx 0.025$} & 04312 & 01856 \\
\hline \multirow[t]{2}{*}{ Feige 11} & $\leq 0.025$ & $39423,34202-4,36961-$ & $3,18543,14018-20,16303-5$ \\
\hline & & 39599,03736 & 18720,03319 \\
\hline Feige 65 & $\leq 0.025$ & 21374 & 02149 \\
\hline Feige 108 & $\leq 0.05$ & 17012,17285 & 13288,13548 \\
\hline Feige 110 & $\leq 0.025$ & 21892 & 02506 \\
\hline HD 4539 & $\leq 0.025$ & 26276 & 07169 \\
\hline HD 149382 & $\leq 0.025$ & $27583,27620,20343$ & 07581,16265 \\
\hline HZ 3 & $0.17 \pm .03$ & $03805,06119,06120$ & 03386,05301 \\
\hline HZ 44 & $\leq 0.025$ & $27240,27242-3$ & 07275,07277 \\
\hline \multicolumn{2}{|c|}{ LSIV $-14116 \leq 0.025$} & 31029 & 10814 \\
\hline \multicolumn{2}{|l|}{ LSIV+00 21} & 37610 & \\
\hline LSIV +109 & $0.05 \pm .03$ & 14813 & 11413 \\
\hline \multicolumn{2}{|c|}{ PG $0342+0260.07 \pm .03$} & 27467 & 07462 \\
\hline \multicolumn{2}{|c|}{$P G 0856+121 \leq 0.025$} & 23159 & 03484 \\
\hline \multicolumn{2}{|l|}{ PG $2111+023$} & 41574 & \\
\hline \multicolumn{2}{|l|}{ PG 2158+082 } & 33793 & \\
\hline \multicolumn{2}{|c|}{ PG $2318+2390.05 \pm .03$} & 42339 & 21099 \\
\hline \multicolumn{2}{|c|}{$\mathrm{PG} 2321+2140.1 \pm .03$} & 39208 & 18329 \\
\hline PHL 25 & \multicolumn{2}{|c|}{$0.025 \pm .0223352$} & 03658 \\
\hline PHL 678 & $\approx 0.2$ & 39286 & 18429 \\
\hline PHL 2726 & $\leq 0.025$ & 40520 & 19505 \\
\hline TON 209 & $\leq 0.025$ & 03722 & 03302 \\
\hline TON 245 & & 30874,28889 & \\
\hline \multicolumn{4}{|c|}{ known or suspected hot sd binaries } \\
\hline BD-3 5357 & \multicolumn{2}{|c|}{$0.05 \pm 0.0233871$} & 13577 \\
\hline BD-7 5977 & $\leq 0.025$ & 31030 & 10815 \\
\hline BD-11 162 & $\leq 0.025$ & $14288,01666,04105$ & 03323,03324 \\
\hline \multicolumn{2}{|c|}{$\mathrm{BD}+284211 \leq 0.025$} & $\begin{array}{l}18899,24727,20317 \\
21088,23273\end{array}$ & $\begin{array}{l}17446,14935,16241 \\
16822,17564\end{array}$ \\
\hline \multicolumn{2}{|c|}{$\mathrm{BD}+293070 \leq 0.025$} & 20344 & 16266 \\
\hline HD 45166 & $0.15 \pm .03$ & $\begin{array}{l}18187,20907,17861 \\
17969,18009,42523\end{array}$ & $16725,14102,09705$ \\
\hline HD 185510 & $0.1 \pm .05$ & $\begin{array}{l}29411,29437,32145, \\
32157,32180\end{array}$ & $\begin{array}{l}09281,14637,11937 \\
11947,11972\end{array}$ \\
\hline \multicolumn{2}{|c|}{ PG $2110+1270.1 \pm .03$} & 41573 & 20310 \\
\hline \multicolumn{3}{|c|}{ PG 2219+0920.025 土. 0239206} & 18328 \\
\hline PHL 1079 & $0.025 \pm .02$ & 242338 & 21098 \\
\hline SB 7 & $\leq 0.025$ & 20559 & 16496 \\
\hline
\end{tabular}


A $15^{\prime \prime}$ aperture was again employed with typical exposures of $2-4$ minutes for each filter for single integrations. Averaged $J H$ and $K$ magnitudes are presented in Tables 3, 4 and 5 together with their corresponding $1 \sigma$ counting-statistic uncertainties. Typical signal-to-noise ratios are at least 5 , and often higher. IR standard stars were observed nightly for calibration (Kidger et al. 1992) and standard reductions were performed using the ESO Snopir software written by Olivia, Barbier, Schmider \& Bouchet (P. Bouchet, private communication), available at the Instituto de Astrofísica de Canarias.

The observations were performed in such a way that the $4^{\prime} \times 4^{\prime}$ area around each target, monitored in real time on a TV screen at the telescope's control, was carefully compared with a finder chart. Systematic checking of the accuracy of the coordinates was also carried out. As the CST has a high pointing accuracy, this task was in reality reduced to having confidence in the listed coordinates of the objects; we used the compilation by Kilkenny et al. (1988) in its latest corrected version (Heber, private communication) or original publications, aided by usage of SIMBAD, ADS or others. We have only found very few entry errors: only in one case was a possibility for misidentification encountered and the object has been dropped from this work. In no case was the $15^{\prime \prime}$ photometer aperture contaminated by a field source (to a limiting $V$ magnitude of about 15.5).

Our JHK values are plotted - February 1994, targets are not repeated - in Fig. 1, together with the corresponding broad energy distribution (see Sect. 3) for each object except for the CSPNs BD-13 842 and $\mathrm{BD}+33$ 2642. Whenever several observations exist, only the weighted average (as marked in Tables 3, 4 and 5 ) is plotted and further analyzed. The inverse of the square of the error on single measurements was used as weights in deriving the weighted averages. The error bar for the $K$ values (and in a few cases also for the $J$ or $H$ ones) is large in the following cases and has not been plotted, for ease-of-display purposes: Feige 11, LSIV-14 11, PG 0242+132, PG 1338+481, PG 2102+037, PG 2111+023, PG 2128+146, PG 2158+082, PG 2218+022, PG 2345+241, PG 2352+181, PHL 678, TON 788.

After our October observations were done, we were informed by the telescope staff that an occasionally wrongly positioned filter wheel might have affected part of our observations. This is indeed the case and can be clearly seen in Fig. 1 for all those stars where values in one of the $J H K$ filters (mostly the $H$ one) are very different (mostly lower) from the rest than it should be, given the values for the other two. This problem may then have affected at least the following stars: Feige 108, Feige 110, HZ 1, HZ 44, LSIV-14 11, PG 0122+214, PG 0133+114, PHL 25, and PHL 2726.

Of these stars only Feige 108 met the $2 \sigma J H K$ excess condition (see Sect. 4) and the rest were, therefore, excluded from further analysis. However, potential binary candidates may be hidden among them as seen from their excesses in two of the filters. Special attention should be paid in this regard to $\mathrm{PG} 0122+214$, PG0133+114, PHL 25, and PHL 2726.

In addition, from visual inspection of Fig. 1, it becomes apparent that several stars with large errors associated to their JHK photometry and/or data with unphysical appearance (i.e., which prevent them from fulfilling the $2 \sigma$ condition for their eventual excess), could still make good binary candidates - these being independent from and in addition to those displaying the filter wheel problem mentioned above. At least, that could be the case for BD+28 4211, LSIV+00 21, PG 0215+183, PG 0242+132, PG 0856+121, PG 1409+605, PG 2102+037, $\mathrm{PG} 2111+023, \mathrm{PG} 2158+082, \mathrm{PG} 2159+051$ and $\mathrm{PG}$ $2229+099$. These stars have also not been analysed in the present paper but we suggest that they, together with the 4 objects mentioned earlier, be investigated in the future.

\section{Archival UV and optical data}

In order to complement our $J H K$ photometry, and to be able to perform our broad-energy analysis, we have compiled published optical data and UV data from the same sources described in Paper I (see therein for references). Resulting energy distributions are plotted in Fig. 1.

\subsection{Optical data}

For BD-3 5357 (FF Aqr), Feige 110, HD 4539, PG 0856+121 and the CSPN BD-13 842 - not analysed, the photometric data collected show offsets among the various systems difficult to interpret in terms of observational errors alone. For some of the stars offsets between sets of photometric data suggest that the cause of the differences is not variability in the sources but systematic differences in the observation and reduction techniques employed by the various authors.

\subsection{IUE data}

After their extraction from the NASA/RDAF data base at Greenbelt (Maryland) and from the Unified Low Dispersion Archive (ULDA) (Wamsteker et al. 1989) at LAEFF (Madrid), we list the IUE spectra employed in this work in Table 6 . The spectra were chosen with the same criteria given in Paper I and were also processed as there described. Reddening corrections - using Seaton's (1979) law, were also applied when required, given the $E(B-V)$ estimates shown in Table 6 . We will not discuss line-profile analysis of the $\mathrm{H}+\mathrm{HeII}$ line at $1215 \AA$ available in the low-dispersion $I U E \mathrm{SW}$ spectra collected. 


\section{Extraction of excess fluxes}

Following the same steps as in Paper I, Tables 1 and 2 list the adopted $T_{\text {eff }}$ for the objects in this paper, based on the literature and our analysis shown in Fig. 1 (available on line).

Fig. 1. The detected $J H K$ fluxes, plotted with available UV and optical data on logarithmic scales. Along the horizontal axis wavelength is plotted in units of $\AA$ while the vertical axis shows $\lambda^{4} \times F_{\lambda}$ in units of $\AA^{4} \times \mathrm{erg} / \mathrm{cm}^{2} / \mathrm{s} / \AA$, along with a model that represents the best estimates of $T_{\text {eff }}$. In each frame the object identify is labeled at the bottom. Near the top there is a legend explaining at which wavelength point the model flux was normalized to the observations. A letter code identifies this point; $U B V$ refers to Johnson magnitudes, uvby to Strömgren data, capital letters subscripted with lower case $g$ refer to Geneva bands and "IUE" followed by a number indicates that the $I U E$ flux was used at the given wavelength (see Thejll et al. 1995) for details

For extraction of excess IR fluxes we scale an appropriate model spectrum to some point in the range of photometric data that we believe is unaffected by radiation from the companion. We always pick the point at which we do the normalization as redward as possible since picking a point far into the UV range can lead to practical problems if the far-UV flux is heavily depressed by metal opacities. Usually this depression is most severe on the blue side of $2000 \AA$. The excess in any band, but in particular the $J H$ and $K$ bands is then simply calculated by subtraction of the normalized model flux from the observations. We use the Kurucz (1993) grid of metal-line blanketed model spectra distributed on CD-ROM disks.

Using Kurucz models has obvious advantages over the simple BB analysis of Paper I. However, it also poses two main drawbacks to our study, as follows: i) the maximum effective temperature available is $50000 \mathrm{~K}$; and ii) no He-rich models are available. Both have an impact on our conclusions regarding the claim of excesses (see Table 7) and must be kept in mind when dealing with their interpretation, specially among the hottest $\mathrm{O}$ type sds: BD + 28 4211, PG 2102+037, PG 2158+082 and PG 2352+181. However, none of these four met the $2 \sigma$ requirement to be analysed for excess. From the February, 1994, data the following sdO objects had already been found as binary candidates in Paper I: BD+10 2357, Feige 34, Feige 80, GD 299, HD 113001 and HD 128220. But constraints i) and ii) may lessen the now newly discovered excesses for BD +37 1977 and BD +48 1777. On the other hand and in general, where the excesses mainly rest, at the longer wavelengths, for both i) and ii) the impact is the least, as already argued in Paper I.

In converting observed magnitudes into physical absolute fluxes we use the zero-point definitions tabulated by Zombeck (1990) for the $U B V R I$ and $J H K$ data, while we used Oke \& Gunn (1983) for Greenstein multichannel data and Table 7 (first column) in Heber et al. (1984) for uvby data (see Paper I). In Table 7 we list the extracted $J$ flux for stars with at least $2 \sigma$ significant excesses (in $J H K$ simultaneously), and also the calculated $J-H$ and $J-K$ colors of the excesses.

Using the Bessel \& Brett (1988) tables of $J-H$ and $J-K$ colors we estimate the range of dwarf spectral classes that correspond to the extracted excess colours. These too are shown in Table 7.

We next derive companion classes for the hot subdwarf by fitting a weighted sum of two model spectra (using Kurucz (1993) models) to the collected and observed photometric data. The fitted weights are proportional to the areas of the stars, so after a successful fit the weight ratio is proportional to the ratio of stellar radii squared. The fit also gives the temperature of the best-fitting cool model. The results are shown in Table 8 .

The fitting procedure is a least- $\chi^{2}$ method and in principle tests the fit of all Kurucz (1993) models against all other models in the grid. In practise we restrict the range of the models to fit since it is prohibitively time-consuming to fit all 3576 Kurucz models against each other - see the caption of the table. The hot model should not be chosen freely as some stars have very accurate atmospheric parameters from the literature and we do not wish to stray far from these values - this is a complicated process as some stars have lots of published photometric data, the fitting of which agrees well with literature values, while other stars have little observed data to work with but may have atmospheric parameters based on spectroscopy, while yet other stars have neither good photometric data nor published atmospheric parameters but only a simple estimate of the spectral type from the literature - frequently based on visual inspections of low dispersion spectra during some classification project.

In a few cases (Feige 80 and GD 299), this method yields a best fit Kurucz effective temperature for the hot component discrepant from previous literature determinations.

In Fig. 2 we compare the dwarf class inferred from extracted colors (Table 7) and fitted models (Table 8). We see that the agreement is quite fair although not complete. Of the 28 stars that had companion-type estimates from both methods, we used 21 stars that have limits on the Bessel \& Brett class - i.e., according to notation in Table 7 no "vl" or " $\rightarrow \mathrm{X}$ " ones were included. Of these, only $\mathrm{BD}+293070$ deviates by more than one spectral class.

The conclusion we can draw from this comparison of derived $\log (g)$ limits is that we appear to have 2 methods that are able, when the data is good, to jointly find the spectral type of the companion to within one class in about $75 \%$ of all cases. 


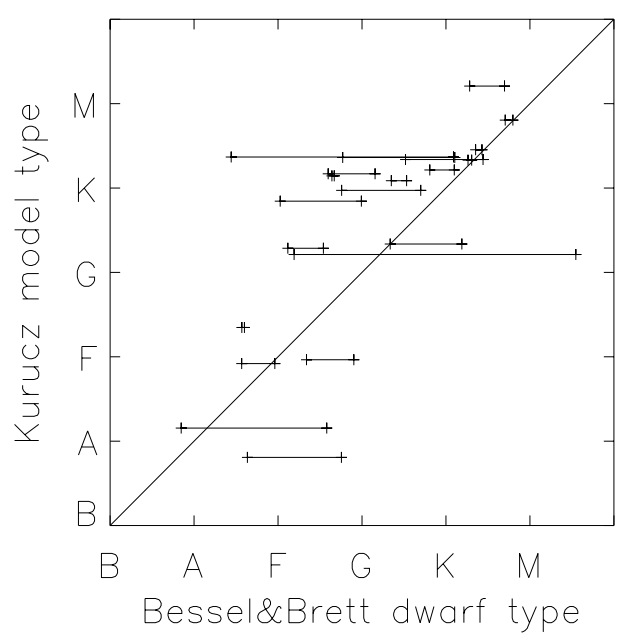

Fig. 2. Comparison of companion types determined by two methods. Along the horizontal axis is plotted the limits on spectral class that can be inferred from the extracted $J-H$ and $J-K$ colours and the Bessel \& Brett (1988) tables for real stars, while the vertical axis displays the spectral type inferred from fits of Kurucz model spectra

\section{Deriving gravities for the hot subdwarfs}

Another result of our model fitting procedure is the determination of gravity estimates for the hot subdwarf, by specifying a mass-gravity-temperature relationship for the companion with the aid of Eq. (5) in Paper I. For purposes of comparing to other work we will use, throughout, the standard assumption of $0.55 M_{\odot}$ (see also Paper I) for the mass of the hot subdwarf.

We can chose whatever mass-gravity-temperature relationship we think is appropriate for the companions, but note that use of a ZAMS relationship will tend to give upper limits on $\log \left(g_{\mathrm{sd}}\right)$ which is desirable, also for comparision purposes. Many companions of evolved luminosity classes have been found and there are selection effects having to do with the relative brightnesses of the two stars that may make sub-giant or giant companions easier to find, in at least optical surveys, but as we use the full range of available UV and our own $J H K$ data we think it is appropriate to at least start with the assumption that most companions will be in their longest phase of evolution - on the MS, and for simplicity we represent the MS by the ZAMS. The ZAMS mass-gravity-temperature relationship we use is based on tabulations such as those in Zombeck (1990). We perform linear interpolations in these tables.

The results of fitting our data is shown in Table 8. For this analysis only data for systems showing a significant excess ( $2 \sigma$ level at least) and yielding a convincingly good fit was employed.
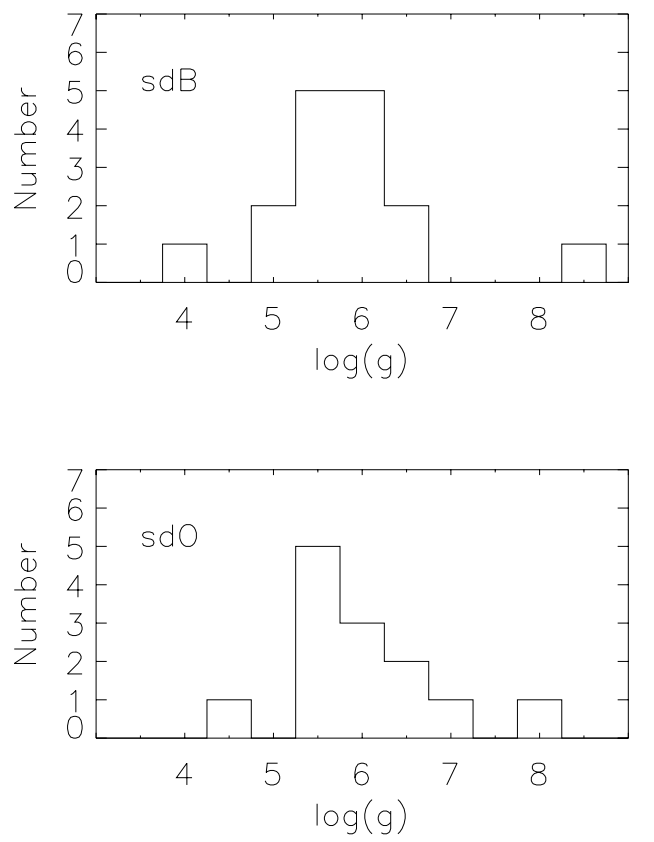

Fig. 3. Derived upper limits for $\log (g)$ of the sample sdB and sdO stars. The bin width is 0.5

We sorted those stars that could be analyzed with the Kurucz model fitting method into the sdB and sdO cases and made histograms of the derived gravity distribution. The results are shown in Fig. 3. The distributions are nearly indistinguishable, given the low numbers involved and that our analysis method is based on assuming ZAMS companions and that if the companion is more luminous for its spectral class than a dwarf then we will overestimate the gravity of the hot subdwarf - the one outlier at high $\log (g)$ in the $\mathrm{sdB}$ case and the 2 highest in the sdO case can then be removed and the difference between the $\log (g)$ distributions is then the slight excess of low gravity cases for the sdB's.

Next, among those stars for which an estimate of $\log (g)$ is available through other means, or for which the luminosity class of the companion is known (Table 8 summarizes what is known), we find a few (i.e., HD 149382, HD 185510, PG2110+127, PHL 1079, Feige 80, GD 299, HD 128220 and MRK 509C) that yield new $\log (g)$ values which may be discrepant by more than about 0.8 from previous ones. Some may contain Giant or Subgiant instead of ZAMS companions; HD 128220, for example, is a well established sd+subgiant case (Howarth \& Heber 1990). We discuss HD 149382 because as a well studied case (Saffer et al. 1994), it provides an example of a contradiction between its known $\log (g)$ values, based on spectroscopic analysis, and our upper limits. Is should be bared in mind the possibility that the cool star in this case be subluminous -i.e., halo system (Heber, private communication). 
All the "binary" cases we have here could of course be cases of chance super-impositions of one star on another with a resulting error in the derived $\log (g)$. "Companion" stars that are closer to us than the hot subdwarf will cause overestimation of the hot subdwarf $\log (g)$, while "companions" that are behind the hot subdwarf will cause the opposite to happen. As super-impositions are not entirely unlikely, but hard to evaluate, it is at present of greater interest to scrutinize our own method, and the individual spectroscopic analyses, in detail. Spectroscopic analyses have, in the contradictory case above, been performed without compensation for the excess flux of the "companion" star. As the super-positioning of stellar spectra can lead to enhancement of spectral lines when lines coincide, and obliteration of lines when the companion star is linefree or -weak, an estimate of the companion spectral class and flux-contribution is needed to evaluate each case.

Several of the stars we have found to be composite have considerable contributions at optical wavelengths from the companion, and we suggest that reanalysis of these spectra, by subtraction of a suitable companion spectrum, could be beneficial in improving the determined atmospheric parameters.

For the particular case of HD 149382, the contribution from the companion to its spectrum of is small - less than $10 \%$ at $5000 \AA$ - so here we do not think the spectroscopic analysis was influenced much by the presence of excess flux. There is no significant discrepancy between the two spectral line analyses carried out by Baschek et al. (1982) with non-LTE models and Saffer et al. (1994) with LTE models for HD 149382 - both results are significantly above the upper limit we get. As the star is bright it is not possible to use various digitized sky surveys to inspect the region near the sky for clues to the origin of the excess flux we see - the size of the aperture we used covers an area entirely dominated by the flux of this star so we can not rule out that the "companion" is rather far from the target and thus unlikely to be physically related. However, as explained in Sect. 2 no visual evidence for contamination was detected at the time of the observation with the CST.

\section{Summary and discussion}

As a continuation of the work reported by Thejll et al. (1995), we have measured the $J H$ and $K$ band fluxes from 72 more stars - mainly hot sdB and sdO stars, along with a few White Dwarfs. In 28 new sd cases (i.e., from June and October, 1994 - see Table 7) we have found a significant excess that we interpret to be mainly due to stellar companions. Of these, 14 are new binary discoveries: BD+25 4655, Feige 108, HD 4539, HD 149382, HD 216135, KPD 2109+440, LSI+63 198, LSIV+10 9, $\mathrm{LSV}+22$ 38, PG 0011+221, PG 0116+242, PG 0314+103, PG 2151+100 and TON 139. BD+25 4655, HD 4539 and
Table 7. The excess fluxes in significant cases. Column 1 gives the object's name, 2 contains the excess $J$ flux and its error, in units of $10^{-24} \mathrm{erg} / \mathrm{cm}^{2} / \mathrm{s} / \mathrm{Hz}$, in 3 and 4 are given the $J-H$ and $J-K$ colours, respectively, of the excess flux. The Bessel \& Brett (1988) dwarf type (their Table II) corresponding to the $J-H$ and $J-K$ colour ranges is in 5 . The overlap in types is given; both type-ranges are shown in all cases where an overlap was not found. "ve" and "vl" indicate ranges that are outside the ranges of the Bessel \& Brett table - either "very early" (i.e. before B8 type) or "very late" (i.e. after M5). The use of the $\rightarrow \mathrm{X}$ notation indicates a range including all previous spectral type up to the $\mathrm{X}$ type. Only systems where the excesses were significant at the $2 \sigma$ level in each of the $J, H$ and $K$ bands are included. As the analysis of individual stars in principle is independent of that in Paper I we repeat objects here that appeared first in Paper I; February 1994, objects are quoted with "F" after the target's name

Name J Jerror $J-H \quad J-K \quad$ type

\begin{tabular}{lll}
$(1)$ & $(2)$ & $(3)$ \\
\hline hot sds/sdBs previously unknown as binaries
\end{tabular}

HD $45390.07+029-0.28 \pm .52$

HD 149382

$0.16 \pm .45 \mathrm{~B} 8-\mathrm{F} 5$

HD 216135

$0.78 \pm .080 \quad 0.38 \pm .12$

$.0 .24 \pm .035-0.51+25-0.60 \pm .42$

$\begin{array}{lllll}\mathrm{KP} 2109+440 & 1.55 \pm .073 & 1.16 \pm .05 & 1.47 \pm .05 \quad \mathrm{vl}\end{array}$

$\mathrm{PG} 0011+221 \quad 0.08 \pm .015-0.15 \pm .24-0.49 \pm .38 \quad$ ve

$\begin{array}{llll}\mathrm{PG} 0116+242 & 0.64 \pm .014 & 0.36 \pm .03 & 0.48 \pm .06 \text { G5-G6 }\end{array}$

$\begin{array}{llll}\mathrm{PG} 0314+103 & 0.14 \pm .018 & 0.66 \pm .14 & 0.94 \pm .16 \quad \mathrm{~K} 6 \rightarrow\end{array}$

$\begin{array}{lllll}\mathrm{PG} 2151+100 & 0.14 \pm .011 & 0.82 \pm .08 & 1.23 \pm .09 & \mathrm{vl}\end{array}$

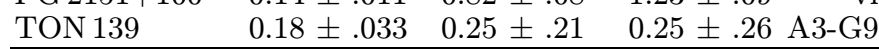

hot sdOs previously unknown as binaries

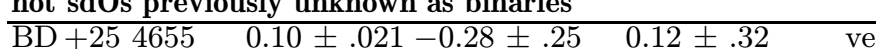

$\mathrm{BD}+371977 \mathrm{~F} \quad 0.12 \pm .044 \quad 0.05 \pm .46-0.09 \pm .49 \rightarrow \mathrm{G} 4$

$\mathrm{BD}+481777 \mathrm{~F} \quad 0.11 \pm .030-0.42 \pm .38-0.78 \pm .42 \quad$ ve

$\begin{array}{llll}\mathrm{LSI}+63198 & 19.79 \pm .366 & 0.66 \pm .02 & 0.80 \pm .03 \mathrm{~K} 6-\mathrm{K} 8\end{array}$

$\begin{array}{lrlll}\mathrm{LSIV}+109 & 0.46 \pm .016 & 0.23 \pm .07 & 0.34 \pm .07 & \mathrm{~F} 4-\mathrm{F} 9\end{array}$

$\begin{array}{llll}\mathrm{LSV}+2238 & 7.64 \pm .001 & 0.37 \pm .01 & 0.57 \pm .01 \mathrm{G} 6 \mathrm{~K} 1\end{array}$

known or suspected hot sd/sdB binaries

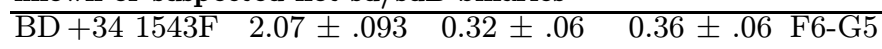

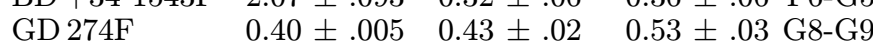

$\begin{array}{llll}\text { HD } 185510 & 55.64 \pm .001 & 0.65 \pm .01 & 0.79 \pm .01 \mathrm{~K} 6-\mathrm{K} 7\end{array}$

$\begin{array}{lllll}\mathrm{HDE} 283048 \mathrm{~F} & 3.40 \pm .201 & 0.22 \pm .08 & 0.29 \pm .08 & \mathrm{~F} 2-\mathrm{F} 9\end{array}$

$\begin{array}{lllll}\text { PB } 8555 & 0.44 \pm .018 & 0.26 \pm .05 & 0.41 \pm .06 & \text { F8-G1 }\end{array}$

$\begin{array}{llll}\mathrm{PG} 0110+262 \mathrm{~F} & 0.15 \pm .027 & 0.09 \pm .25 & 0.24 \pm .30 \rightarrow \mathrm{G} 4\end{array}$

$\begin{array}{lllll}\mathrm{PG} 0232+095 \mathrm{~F} & 0.56 \pm .073 & 0.34 \pm .15 & 0.50 \pm .17 \quad \mathrm{~F} 6-\mathrm{K} 1\end{array}$

$\begin{array}{lllll}\text { PG } 2110+127 & 0.18 \pm .011 & 0.23 \pm .09 & 0.19 \pm .11 & \text { F0-F5 }\end{array}$

$\begin{array}{llll}\mathrm{PG} 2118+126 & 0.12 \pm .014 & 0.04 \pm .16 & 0.08 \pm .22\end{array} \rightarrow \mathrm{F} 3$

PG 2148+095 $0.18 \pm .022-0.18 \pm .23 \quad 0.18 \pm .42 \rightarrow \mathrm{A} 4$

$\begin{array}{llll}\mathrm{PG} 2219+094 & 0.11 \pm .018 & 0.01 \pm .19 & 0.02 \pm .27\end{array} \rightarrow \mathrm{F} 3$

$\begin{array}{llll}\text { PHL } 1079 & 0.13 \pm .025 & 0.38 \pm .19 & 0.60 \pm .20 \text { G4-K3 }\end{array}$

$\begin{array}{llll}\text { SB } 7 & 0.08 \pm .037 & 0.55 \pm .41 & 0.44 \pm .45 \text { F0-M4 }\end{array}$

known or suspected hot sdO/sdOB binaries

\begin{tabular}{lllll}
\hline $\mathrm{BD}-35357$ & $20.79 \pm .193$ & $0.53 \pm .01$ & $0.68 \pm .01$ & $\mathrm{~K} 3$
\end{tabular}

$\begin{array}{lrll}\mathrm{BD}-75977 & 4.13 \pm .079 & 0.50 \pm .02 & 0.61 \pm .02 \mathrm{~K} 1-\mathrm{K} 2\end{array}$

$\begin{array}{llll}\text { BD-11 } 162 & 0.58 \pm .022 & 0.25 \pm .05 & 0.31 \pm .05 \quad \mathrm{~F} 4-\mathrm{G} 0\end{array}$

$\mathrm{BD}+102357 \mathrm{~F} \quad 6.08 \pm .182 \quad 0.11 \pm .05 \quad 0.11 \pm .05$ A5-F1

$\mathrm{BD}+293070 \quad 1.60 \pm .019 \quad 0.26 \pm .02 \quad 0.28 \pm .04 \quad \mathrm{~F} 5-\mathrm{F} 6$

$\begin{array}{lllll}\text { Feige } 34 \mathrm{~F} & 0.09 \pm .016 & 0.79 \pm .21 & 0.79 \pm .21 & \mathrm{~K} 3 \rightarrow\end{array}$

$\begin{array}{lllll}\text { Feige 80F } & 0.39 \pm .056 & 0.30 \pm .16 & 0.19 \pm .17 & \text { F0-F9 }\end{array}$

$\begin{array}{llll}\text { GD 299F } & 0.13 \pm .039 & 0.20 \pm .33 & 0.20 \pm .32 \rightarrow \text { G9 }\end{array}$

$\begin{array}{lllll}\text { HD } 45166 & 0.93 \pm .044 & 0.08 \pm .07 & 0.30 \pm .09 & \text { A5 F7 }\end{array}$

$\begin{array}{lllll}\mathrm{HD} 113001 \mathrm{~F} & 3.06 \pm .065 & 0.11 \pm .03 & 0.06 \pm .03 & \mathrm{~A} 5\end{array}$

$\begin{array}{llll}\text { HD } 128220 \mathrm{~F} & 17.38 \pm .340 & 0.32 \pm .03 & 0.41 \pm .02 \text { G3-G5 }\end{array}$

$\begin{array}{lrll}\text { MRK509C } & 0.07 \pm .025 & 0.23 \pm .39 & 1.00 \pm .40 \mathrm{~K} 2-\mathrm{K} 5\end{array}$


HD 216135 are however the weakest cases as their evidence for an IR excess becomes marginal when compared to other clearer cases. Two more discoveries are BD +37 1977 and BD +48 1777 which, from Paper I, are now found to display excesses with our present analysis. It should be kept in mind, however, that both are sdO cases now fitted with H-rich Kurucz models which, together with the previous marginal evidence for an excess pointed out in Paper I, render this conclusion weak as well. BD-13 842 and BD+33 2642 display also IR flux escesses but, being classified as CSPN, have not been analysed. The suspected binaries PB 8555 (Kilkenny et al. 1988) and SB 7 (Heber 1986) are confirmed. Our two analysis methods (extraction of $2 \sigma$ simultaneously significant $J H K$ flux excesses and fitting of two Kurucz atmospheric models) can jointly find agreeing spectral type companions for 21 out of 28 "good data" (see Table 8) cases. 13 companions could be of G type (see Table 7).

The 28 analysed new cases, added to the 11 sds found by Thejll et al. (1995), represent $44 \%$ out of the 88 hot sds observed in total during 1994 (i.e., $=24$ from February +64 from June and October). Of the 41 (i.e., $=28+11+\mathrm{BD}+371977+\mathrm{BD}+48$ 1777), fulfilling a $2 \sigma$ excess condition, 15 are sdO, 15 are sdB, three are sdOBs, $\mathrm{PG} 0110+262$ is an $\mathrm{sdB}-\mathrm{O}$ and the rest (i.e., 7) remain unclassified (i.e., sd).

For 28 stars (see Table 8) we were furthermore able to satisfactorily fit the sum of two spectral Kurucz models so that temperature estimates and an estimate of the relative radii could be obtained. Assuming that all the companions are ZAMS stars we can then calculate upper limits on $\log (g)$ for the hot subdwarfs. For 18 of the hot subdwarfs previous estimates of $\log (g)$ were available from spectroscopic analysis and our upper limits are in agreement with most cases except for a few discrepant ones. We discuss HD 149382 as an exaple which we find inexplicable, as the excess flux is small and thus probably did not influence the several spectroscopic analysis that have been performed - we speculate that the source of the excess radiation is unrelated to the star itself, but cannot evaluate this suggestion without better imaging of possible faint sources in the sky around the bright main star. Other discrepant cases may be due to the presence of Giant or Subgiant, instead of ZAMS, companions.

Given the recent discovery of pulsators among hot subdwarfs with binary companions (O'Donoghue et al. 1997 and references therein) our sample provides suitable candidates to pursue that line of investigation. We are currently investigating, both theoretically and observationally, the presence of eventual pulsations in Feige 108 among others.

\subsection{Gravities and evolutionary implications}

We have also found that the distributions of the derived upper limits of $\log (g)$ are very similar for the sdO and
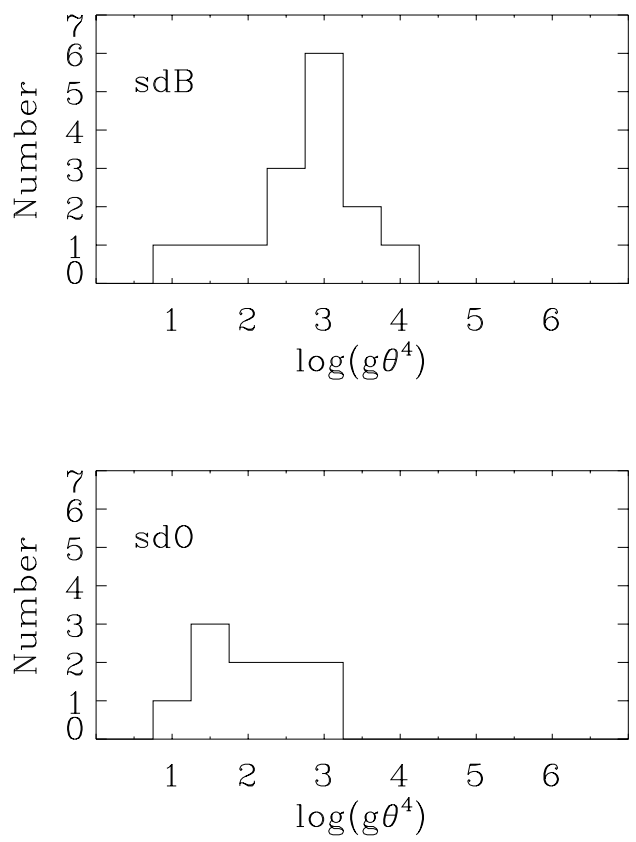

Fig. 4. Derived upper limits for $\log \left(g \theta^{4}\right)$ of the sample sdB and sdO stars. Stars with known giant companions have been excluded

sdB cases - both show a substantial concentration of cases between about 5.25 and 6.5 in $\log (g)$. Direct spectroscopic determination of atmospheric parameters for sdB and $\mathrm{sdO}$ stars usually give distributions of $\log (g)$ for sdBs between 5.3 and 6.2 with sdOs distributed over a similar range but now and then, depending on the sample of stars, going down to $\log (g)=4.1$ (e.g. HD 128220).

We had hoped to find several telling cases of sdOs with companions so that we could "test" NLTE modelling techniques, but we have found no strongly contradictory cases within the present analysis.

As our method only provides upper limits on $\log (g)$ for the hot star, the narrowness of the $\log (g)$ distributions can be interpreted to imply that the companion-types are all well described by our assumption of ZAMS status real giant or sub-giant companions force our method to exaggerate the upper limits on $\log (g)$ but the absence of a substantial number of cases like that shows the predominance of un-evolved companions. This is what one would expect on the basis of probability considerations as most stars spend most of their time in the hydrogen burning phase.

The coincidence of the main peaks in the $\log (g)$ distributions - to the extent that we are able to "resolve" it with the limited number of stars we have - may say something about the underlying mass-distributions. Saffer et al. (1994) found that the distribution of $\log \left(g \theta^{4}\right)$ (where $\left.\theta=5040 / T_{\text {eff }}\right)$ for the sdB stars they studied was a basically unresolved peak near 2.6 and a FWHM of 0.4 (i.e. 
Table 8. Results of fitting Kurucz models to the observed fluxes and summary of literature data for hot $\operatorname{subdwarf} \log (g)$ values, and/or luminosity classes of companions. The columns are: (1) Object name, (2) $T_{\text {eff }}$ of the assumed or fitted hot model, (3) the $T_{\text {eff }}$ of the fitted cool model, followed in parenthesis by the dwarf spectral type derived from the temperature and standard tables (Zombeck 1990), (4) The ratio $R^{\prime}=R$ (cool star) $/ R$ (hot sd), (5) the derived gravity of the hot subdwarf (upper limit) calculated from an assumed sd mass of $0.55 M_{\odot}, R^{\prime}$ and a mass of the cool companion derived from the mass-radius- $T_{\text {eff }}$ relationship for ZAMS stars from Zombeck, (6) literature values for surface gravity (with reference in parenthesis, see Table 1 and below) and (7) luminosity class of the companion from the literature, if known. In all cases, except for BD+34 1543 ([-0.5]), we have used solar metallicity models. Whenever possible we have chosen the $\log (g)=5.0$ model for the hot star. Notes: ${ }^{a}=\log (g)$ based on equivalent widths determination; ${ }^{b}=\log (g)$ based on $R$ and $I$ photometric determinations by Thejll et al. (1994b; Th94). All other $\log (g)$ determinations are spectroscopic. B82=Baschek et al. (1982). B93=Boffin et al. (1993). K88=Kilkenny et al. (1988), Ra93=Rauch (1993), WSp60=Wallerstein \& Spinrad (1960), WSK63=Wallerstein et al. (1963), WW66=Wallerstein \& Wolff (1966), WS83=Willis \& Stickland (1983)

\begin{tabular}{|c|c|c|c|c|c|c|}
\hline $\begin{array}{l}\text { Object } \\
(1)\end{array}$ & $\begin{array}{r}T_{\text {eff }}(1) \\
(2) \\
\end{array}$ & $\begin{array}{r}T_{\text {eff }}(2) \\
(3)\end{array}$ & $\begin{array}{l}R^{\prime} \\
(4)\end{array}$ & $\begin{array}{l}\log (g) \\
(5)\end{array}$ & $\begin{array}{l}\text { lit. } \log (g) \\
(6)\end{array}$ & $\begin{array}{l}\text { L. class of comp. } \\
(7)\end{array}$ \\
\hline \multicolumn{7}{|c|}{ hot sds/sdBs previously unknown as binaries } \\
\hline Feige 108 & 35000. & $5500(\mathrm{G} 6)$ & 4. & 5.5 & & \\
\hline HD 149382 & 34000. & 5750.(G2) & 2.3 & 5.0 & $5.89(\mathrm{~S} 94), 5.5 \pm .3(\mathrm{~B} 82)$ & \\
\hline PG $2151+100$ & 27000 . & 3500.(M2) & 10. & 6.7 & & \\
\hline TON 139 & 18000. & 4750.(K2) & 5. & 5.7 & & \\
\hline \multicolumn{7}{|c|}{ known or suspected hot $\mathrm{sd} / \mathrm{sdB}$ binaries } \\
\hline $\mathrm{BD}+341543$ & 26000. & $5500 .(\mathrm{G} 8)$ & 8. & 6.0 & $5.9(\mathrm{pI})$ & \\
\hline GD 274 & 24000 . & 4750.(K2) & 7. & 6.0 & $6.2(\mathrm{pI})$ & \\
\hline HD 185510 & 25000 . & 4250.(K8) & 164. & 8.9 & $6.0(\mathrm{Je} 92)$ & K0III-IV(K88) \\
\hline HDE283048 & 40000. & 7500.(A8) & 13. & 6.2 & $6.4 \pm .3(\mathrm{pI})$ & \\
\hline PG $0110+262$ & 21000. & 5000.(K1) & 6. & 5.9 & $5.5(\mathrm{pI})$ & \\
\hline PG $0232+095$ & 21000. & 4750.(K2) & 13. & 6.6 & $6.9 \pm .3(\mathrm{pI})$ & \\
\hline PG $2110+127$ & 34000 . & 5750.(G2) & 8. & 5.9 & $5.33(\mathrm{~S} 94), 5.0 \pm .2(\mathrm{~T} 95)$ & G6IV(T95) \\
\hline PG $2118+126$ & 25000. & 5250.(K0) & 7. & 6.0 & & \\
\hline PG $2148+095$ & 25000 . & 5000.(K1) & 6. & 5.8 & & \\
\hline PG $2219+094$ & 21000. & 7000.(F1) & 2.8 & 5.0 & $4.6(\mathrm{~S} 94)$ & \\
\hline PHL 1079 & 30000. & 4750.(K2) & 9. & 6.2 & $5.25(\mathrm{~T} 95)$ & G8IV(T95) \\
\hline SB 7 & 50000. & 5750.(G2) & 7. & 5.9 & & \\
\hline \multicolumn{7}{|c|}{ known or suspected hot sdO/sdOB binaries } \\
\hline $\mathrm{BD}-35357$ & 40000. & $4500 .(\mathrm{K} 4)$ & 67. & 8.1 & & G8III(K88), K0IV-III(B93) \\
\hline $\mathrm{BD}-75977$ & 31000. & 4750.(K2) & 23. & 7.1 & & K0IV-III(V91) \\
\hline $\mathrm{BD}-11162$ & 35000. & 5250.(K0) & 6. & 5.9 & & \\
\hline $\mathrm{BD}+102357$ & 27000. & 5750.(G2) & 7. & 5.9 & $5.6(\mathrm{pI})$ & \\
\hline $\mathrm{BD}+293070$ & 18000. & 5250.(K0) & 4. & 5.5 & & \\
\hline Feige 34 & 50000. & 3500.(M2) & 4.6 & 6.1 & $6.8_{-0.7}^{+0.3}(\mathrm{pI}, \operatorname{trig} \pi)$ & \\
\hline Feige 80 & 33000. & 5500.(G8) & 6. & 5.7 & $4.83(\mathrm{~S} 94)$ & \\
\hline GD 299 & 37500 . & 4750.(K2) & 5.0 & 5.8 & $4.9^{a}(\mathrm{GS} 74)$ & \\
\hline HD 45166 & 40000. & 13000.(B8) & 7. & 4.8 & $4.4(\mathrm{WS} 83)$ & B8V(WS83) \\
\hline HD 113001 & 47500 . & 6750.(F3) & 9. & 6.0 & $5.6-5.9(\mathrm{To} 70), 6.0(\mathrm{pI})$ & F2V(WSp60) \\
\hline HD 128220 & 40000. & 5250.(K0) & 16. & 6.7 & 4.1(Ra93) & Giant(WSK63; WW66) \\
\hline MRK509C & 42500. & 3500.(M2) & 8. & 6.6 & $5.0-5.3^{b}(\mathrm{Th} 94)$ & \\
\hline
\end{tabular}

standard deviation near 0.2 ). This corresponded to a single Gaussian mass distribution with a narrow width of $1 \sigma \sim 0.04 M_{\odot}$ centered on $0.5 M_{\odot}$. As $g \theta^{4}$ scales with luminosity and is constant for a given core mass (Greenstein \& Sargent 1974) it is consistent to interpret the distribution found by Saffer et al. as evidence for core He burning. In our sample of stars we find (after excluding the known cases of giant companions) that the distributions of $\log \left(g \theta^{4}\right)$ have mean and standard deviations of $(2.9$; $0.8)$ and $(2.3 ; 0.7)$ for the sdBs and sdOs, respectively. The distributions are shown in Fig. 4.
These values are not significantly different from each other, nor from the more narrowly distributed value that Saffer et al. found for their sample of sdBs. Analysis of the mass-luminosity ratio for helium-core burning objects (Thejll \& MacDonald, unpublished models), shows that near $0.5 M_{\odot}$ a doubling or halving of the mass corresponds to an order of magnitude decrease or increase in the $\mathrm{M} / \mathrm{L}$ ratio, respectively. As the present distribution of $\log \left(g \theta^{4}\right)$ for the sdOs contains most of the cases within one order of magnitude on either side of the mean we see that the distribution corresponds to a spread in masses for the sdOs 
between 0.25 and $1 M_{\odot}$, if they are assumed to be helium core burners. These are not unrealistic limits given what we know about the mass distribution of sdOs from suitable binary systems (Ritter 1990). The present data on the sdOs is therefore consistent with an interpretation of the sdOs as being He-core burners (i.e. Helium Main Sequence stars - HEMS) with a natural spread of masses.

Future improvements in the amount and quality of data available for sdOs will be needed to make a statement about whether a narrower distribution of masses such as in a scenario where sdBs evolve into sdOs, and therefore keep their narrowly distributed mass-spectrum - is the case.

Acknowledgements. Peter Thejll gratefully acknowledges support from Nordita and the Danish Natural Research Council. Ana Ulla acknowledges support from the Spanish DGICYT for a contract under the MEC's programme "Incorporación de Doctores y Tecnólogos a grupos de Investigación Científica y Enseñanza Superior" associated to CICYT's research project ISOPHOT-S Fase Final (contract ESP94-0034), the EU (contract ERBCHBGCT930407) and Nordita for visiting grants in May and October, 1996, to work on this paper. Staff at the Carlos Sánchez Telescope are thanked for their skilled assistance during observations. C.S.Hansen is also thanked for obtaining for us some of the October, 1994, observations. This research has made use of the SIMBAD database, operated at CDS, Strasbourg, France and of the NASA's Astrophysics Data System (ADS). Use has also been made of the RDAF facility at the GSFC at Greenbelt Maryland and of the ULDA at the LAEFF in Madrid (Spain). Henrik Svensmark is thanked and admired for his insight into IDL font magics. Ana Ulla is an external scientific collaborator of the Laboratorio de Astrofísica Espacial y Física Fundamental in Madrid (Spain).

\section{References}

Allard F., Wesemael F., Fontaine G., Bergeron P., Lamontagne R., 1994, AJ 107, 1565

Arribas S., Martinéz-Roger C., 1987, A\&A 70, 303

Baschek B., Kudritzki R.P., Scholz M., Simon K.P., 1982, A\&A 108, 387

Bauer F., Husfeld D., 1995, A\&A 300, 481

Bessel M., Brett J.M., 1988, PASP 100, 1134

Boffin H.M.J., Cerf N., Paulus G., 1993, A\&A 271, 125

Bowyer, et al., 1994, ApJS 93, 569

Dreizler S., 1993, A\&A 273, 212

Dreizler S., Heber U., Werner K., Moehler S., de Boer K.S., 1990, A\&A 235, 234

Giddings J.R., 1980, Ph.D. thesis, Univ. Coll. London

Greenstein J.L., Sargent A.I., 1974, ApJS 28, 157

Heber U., 1986, A\&A 155, 33

Heber U., Hunger K., Kudritzki R.P., Simon K.P., 1984, in Maeder and Renzini (eds.) "Observational tests of the stellar evolution theory" IAU, p. 215
Heber U, Langhans X., 1986, in "New Insigths in Astrophysics", ESA SP-263, p. 279

Howarth I.D., Heber U., 1990, PASP 102, 912

Hurwitz M., Bowyer S., 1995, ApJ 446, 812

Jeffery J.S.E., Simon K.P., Evans 1992, MNRAS 258, 64

Jiménez R., et al., 1994, in "Hot stars in the Galactic Halo", Adelman, Upgren \& Adelman (eds.) CUP, p. 211

Kidger M., et al., 1992, IAC-CST Technical Note 16

Kilkenny D., Heber U., Drilling J., 1988, SAAO Circ. No. 12 , and later electronic versions thereof distributed by the authors

Kurucz R.L., 1993, Kurucz CD-ROM No. 13

Marilli E., Frasca A., Bellina Terra M., Catalano S., 1995, A\&A 295,393

Moehler S., et al., 1990, A\&A 239, 265

Napiwotzki R., 1993a, Acta Astron. 43, 343

Napiwotzki R., 1993b, Acta Astron. 43, 415

O'Donoghue D., Lynas-Gray A.E., Kilkenny D., Stobie R.S., Koen C., 1997, MNRAS 285, 657

Oke J.B., Gunn J.E., 1983, ApJ 266, 713

Peterson, D., 1970, Ph.D. thesis Caltech

Probst R., 1983, ApJSS 53, 335

Rauch T., 1993, A\&A 276, 171

Ritter H., 1990, A\&AS 85, 1179

Saffer R., Bergeron P., Koester D., Liebert J., 1994, ApJ 432, 351

Seaton M., 1979, MNRAS 187, 73p

Theissen A., Moehler S., Heber U., de Boer K.S., 1993, A\&A 273,524

Theissen A., Moehler S., Heber U., Schmidt J.H.K., de Boer K.S., 1995, A\&A 298, 577

Thejll P., Bauer F., Saffer R., Liebert J., Kunze D., Shipman H.L., 1994a, ApJ 433, 819

Thejll P., Flynn C., Williamson R., Saffer R., 1997, A\&A 317, 689

Thejll P., Jiménez R., Saffer R., Jørgensen U.G., 1994b, in proceedings of the "Hot stars in the Halo" conference held at Schenectady NY, U.S.A., Adelman S.J., Upgren A.R., Adelman C.J. (eds.). Cambridge University Press

Thejll P., Ulla A., MacDonald J., 1995, A\&A 303, 773

Tomley L., 1970, ApJ 162, 239

Vennes S., Dupuis J., Rumph T., Drake J., Bowyer S., Chayer P., Fontaine G., 1993, ApJ 410, L119

Vennes S., Thorstensen J.R., 1994, AJ 108, 1

Vennes S., Ulla A., Thejll P., 1998 (in preparation)

Viton M., Deleuil M., Tobin W., Prevot L., Bouchet P., 1991, A\&A 242, 175

Wallerstein G., Spinrad H., 1960, PASP 72, 486

Wallerstein G., Sturch G., Klemola A.R., 1963, PASP 75, 61

Wallerstein G., Wolff S.C., 1966, PASP 78, 390

Wamsteker W., et al., 1989, A\&AS 79, 1

Willis A.J., Stickland D.J., 1983, MNRAS 203, 619

Wolff B., Jordan S., Koester D., 1996, A\&A 307, 9

Zombeck M.V., 1990, Handbook of Space Astronomy \& Astrophysics. Cambridge, Cambridge University Press, 2nd ed. 